\title{
Continuous postoperative negative pressure irrigation assisted mammaplasty in treating chronic refractory plasma cell mastitis
}

\author{
Hua Xu ${ }^{1 \#}$, Yan Jiang ${ }^{2 \#}$, Mingjuan $\mathrm{Liao}^{3}$, Dongliang $\mathrm{Li}^{4}$, Chenfang $\mathrm{Zhu}^{1}$ \\ ${ }^{1}$ Department of General Surgery, Shanghai Ninth People's Hospital, Shanghai Jiao Tong University School of Medicine, Discipline Construction \\ Research Center of China Hospital Development Institute, Shanghai Jiao Tong University, Shanghai, China; ${ }^{2}$ Department of Thoracic Surgery, \\ Shanghai Pulmonary Hospital, Tongji University School of Medicine, Shanghai, China; ${ }^{3}$ Department of Traditional Chinese Medicine, Shanghai \\ Ninth People's Hospital, Shanghai Jiao Tong University School of Medicine, Discipline Construction Research Center of China Hospital \\ Development Institute, Shanghai Jiao Tong University, Shanghai, China; ${ }^{4}$ Department of Thoracic Surgery, Shanxi Provincial Tumor Hospital, \\ Taiyuan, China \\ Contributions: (I) Conception and design: C Zhu, H Xu, DL Li; (II) Administrative support: C Zhu; (III) Provision of study materials or patients: All \\ authors; (IV) Collection and assembly of data: All authors; (V) Data analysis and interpretation: All authors; (VI) Manuscript writing: All authors; (VII) \\ Final approval of manuscript: All authors. \\ "These authors contributed equally to this work. \\ Correspondence to: Chenfang Zhu. Department of General Surgery, Shanghai Ninth People's Hospital, Shanghai Jiao Tong University School of \\ Medicine, Discipline Construction Research Center of China Hospital Development Institute, Shanghai Jiao Tong University, Shanghai 200011, \\ China. Email: sammizz1977@126.com; Dongliang Li. Department of Thoracic Surgery, Shanxi Provincial Tumor Hospital, Taiyuan 030013, China. \\ Email: sxzlyyxw@126.com.
}

Background: Chronic refractory plasma cell mastitis (CRPCM) is an aseptic inflammation of the breast with a chronic course of the disease, extended treatment cycle (months to years), with a high recurrence rate. The objective of this study was to evaluate the efficacy of continuous postoperative negative pressure irrigation assisted mammaplasty (CPNPIAM) in treating CRPCM.

Methods: Between 2016 and 2018, 36 CRPCM patients receiving CPNPIAM were enrolled in this study. CPNPIAM mainly involved complete lesion removal, immediate breast mammaplasty, and continuous postoperative negative pressure irrigation. The age of the patients, local symptoms, history of treatment, the duration of the disease before surgery, hospitalization period, related risk factors, the success rate, the recurrence rate and patients' overall satisfaction ratings were analyzed in the article.

Results: Patients were aged between 22 and 53 years (mean 34.64 years). All patients had a history of conservative treatment or simple drainage. Local symptoms included inflammatory mass $(\mathrm{n}=36,100 \%)$, abscess $(n=33,92 \%)$, nipple discharge $(n=7,19 \%)$, inflammatory plaque $(n=34,94 \%)$, and sinus tract formation $(\mathrm{n}=19,53 \%)$. The lesion sizes ranged from 3 to $10 \mathrm{~cm}$ (mean $5.13 \mathrm{~cm})$ in diameter. The mean hospitalization period was 8.42 days. The success rate was $100 \%(36 / 36)$ and the recurrence rate was $0 \%$ $(0 / 36)$ at a 3 -month follow-up. The patients' overall satisfaction ratings were "very good" ( $n=22,61 \%)$, "good" $(\mathrm{n}=12,33 \%)$, and "moderate" $(\mathrm{n}=2,6 \%)$ with no poor or unsatisfactory ratings.

Conclusions: CPNPIAM is an effective way of treating CRPCM, and showed a high success rate, a low recurrence rate, and high patient satisfaction.

Keywords: Chronic refractory plasma cell mastitis (CRPCM); surgery; mammaplasty; continuous postoperative negative pressure irrigation

Submitted Sep 27, 2020. Accepted for publication Dec 17, 2020.

doi: 10.21037 /gs-20-795

View this article at: http://dx.doi.org/10.21037/gs-20-795 


\section{Introduction}

Plasma cell mastitis (PCM) is a benign non-infectious inflammatory breast disease characterized by dilation of the mammary ducts and infiltration of plasma cells and was first reported by Cheatle and Cutler in 1949 (1). PCM normally occurs in young and middle-aged women who are not lactating or pregnant. PCM is challenging to be treated, and the recurrence rate is high $(2,3)$. Some studies have reported the incidence of PCM in benign breast disease as $4.1-5.5 \%$. However, other researchers have reported a higher incidence rate of PCM in recent years $(4,5)$. The etiology of PCM is still unclear. Risk factors include the following: a congenitally inverted nipple, breast dysplasia, milk retention, trauma, surgical infections, autoimmune diseases, endocrine hormone disorders, and smoking $(6,7)$. There are differences between the acute and chronic phases of PCM. In the acute phase, PCM presents as acute inflammation of breast with reddish skin that is swollen, hot, and painful. PCM with a course of disease greater than one month is defined as chronic plasma cell mastitis. Repeated treatment failure or recurrence in patients with chronic plasma cell mastitis is defined as chronic refractory plasma cell mastitis (CRPCM). The clinical manifestations of CRPCM mainly involve nipple retraction, nipple discharge, inflammatory mass, abscess, and sinus tract formation (3,5-8). Breast inflammatory mass mostly locates in the areola area, often accompanied by nipple retraction with or without nipple discharge. CRPCM can also present ipsilateral axillary lymph node enlargement. Normally, patients do not display a systemic inflammatory response, and antibiotics are ineffective. Typical syndrome, ultrasound and MRI examination and pathological examination by core needle biopsy are necessary for the diagnosis of CRPCM. Pathological examination of CRPCM is characterized by dilation of the mammary ducts and infiltration of plasma cells.

The current treatment of PCM is complex, with a high recurrence rate $(8,9)$. Chinese medicine, dexamethasone, tamoxifen and anti-tuberculosis drugs have been recommended for the treatment of plasma cell mastitis. However, the effects were limited. Surgery remains to be the most effective treatment. Traditional surgical procedure for PCM mainly involves repeated simple drainage, lumpectomy, quadrant resection, or mastectomy, which is still subject to recurrence and breast deformity. Some patients experience physical or psychological distress due to the repeated treatment failure, long disease course, recurrence, breast deformity or breast absence (4-10). Therefore, it is critical to find an ideal treatment regimen for CRPCM. This article reports on a novel surgical technique CPNPIAM for treating CRPCM. The procedure of CRPCM involves complete lesion removal, immediate breast mammaplasty, and continuous postoperative negative pressure irrigation. Satisfactory results were achieved, as reported below.

We present the following article in accordance with the STROBE reporting checklist (available at http://dx.doi. org/10.21037/gs-20-795).

\section{Methods}

\section{Patients}

A total of 36 patients who received CPNPIAM in our hospital between November 2016 and October 2018 were retrospectively included in this study. All patients were diagnosed as CRPCM according to typical syndromes, disease course, ultrasound, MRI, and pathological examination by cord needle biopsy before surgery. Pathological examination was verified by two experienced pathologists. The images of MRI before CPNPIAM are shown in Figure 1. The study was approved by Shanghai Ninth People's Hospital, Shanghai Jiao Tong University School of Medicine institutional ethical committee standards (institutional ethical committee number: 2016194-T138). All patients signed an informed consent form. The study conformed to the provisions of the Declaration of Helsinki (as revised in 2013).

\section{Case selection criteria}

The inclusion criteria were as follows: (I) female gender; (II) with a course of illness longer than one month; and (III) experienced unsuccessful conservative treatment or repeated simple drainage, lumpectomy. Exclusion criteria were as follows: (I) male gender; (II) with suspected breast cancer or mastitis of other causes; and (III) with a course of illness less than 3 months.

\section{Surgical technique}

Before the surgery, the excision area was drawn on the skin with the patient in the standing position (Figure $2 A, B, C$ ). Under general anesthesia, the patient was placed in a supine position with both arms outstretched. If necessary, the 

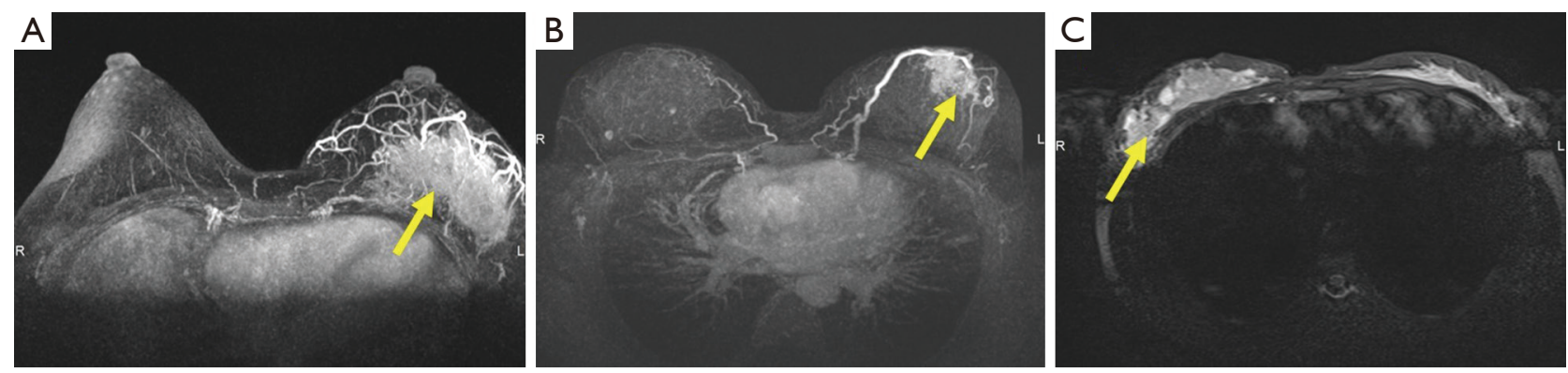

Figure 1 Magnetic resonance imaging (MRI) of plasma cell mastitis (PCM) lesion before surgery. (A,B,C) MRI images of PCM lesion (arrow) before surgery in three patients with chronic refractory plasma cell mastitis.

operating table was adjusted in a position better to observe the cosmetic appearance of the bilateral breasts. The skin should be kept as much as possible, except the sinus area or necrotic tissue. All inflammatory masses, abscesses, sinus tract formations, and abnormal mammary ducts were thoroughly resected. The surrounding breast tissue should be carefully checked to ensure no lesions residue (Figure $2 D$ ). The excised area was washed with hydrogen peroxide $(250 \mathrm{~mL})$ and saline solution $(1,000 \mathrm{~mL})$. The glandular flaps originating from the surrounding breast tissue was separated and rotated to the excised area for immediate breast mammaplasty (Figure 2E). The position of nipple areola was adjusted by removing dermis around areola. Locally advanced skin flap was designed to cover the absence of skin, according to local defect situation. A negative pressure suction equipment was arranged into the surgical area for continuous postoperative negative pressure irrigation with negative pressure $0.02 \mathrm{mmHg}$ (Figure $2 F$ ). The saline irrigation solution flowing from the tube's entrance point drains the exudates and inflammatory factors of the wound out via the tube's exit point, which is connected to the vacuum drainage system. After 3-7 days of continuous saline irrigation, the drainage tube was removed before the patient was discharged from hospital. The appearances of the breast on post-operative Day 7 (Figure 2G) and 6-month (Figure 2H) were recorded. The equipment of continuous postoperative negative pressure irrigation which includes saline irrigation, multiple-hole drainage tube and vacuum system is shown in Figure 3.

\section{Clinical assessment}

Clinical assessment measures including age, history of treatment, disease duration before surgery, local symptoms, hospitalization time and related risk factors were assessed and documented after surgery. The success rate, recurrence rate, and patient satisfaction ratings were assessed after a 3-month follow-up. Patients were asked by the investigator, using Breast-Q tables, to evaluate their degree of satisfaction (very good, good, moderate, poor, failed) regarding the overall procedure, the shape, size, and symmetry of their breasts, and the incidence of recurrence. Images comparing the appearance and shape of the breasts before and after surgery are shown in Figure 4.

\section{Statistical analysis}

Continuous variables were presented as mean \pm standard deviation. Categorical variables were presented as numbers and percentages. All data were calculated with SPSS 22.0 (IBM Corp., Armonk, NY, USA).

\section{Results}

All 36 patients were Chinese females, ranging in age from 22-53 years (mean 34.64 years). CRPCM was unilateral in 34 patients $(94 \%)$ and bilateral in two patients $(6 \%)$. Ten patients were aged between 20-29 years (28\%), 17 patients were aged between $30-39$ years (47\%), six patients were aged between $40-49$ years (17\%), and three patients were aged between $50-59$ years ( $8 \%)$. None of the patients was over 60 years of age (Table 1). All patients had a history of medicine treatment or repeated simple drainage, 18 cases had received antibiotics (50\%), 33 cases had received Chinese medicine (92\%), and 20 cases had received prior more than once time simple drainage $(56 \%)$. The systemic symptoms included 5 patients with low fever (14\%), while 31 patients demonstrated no systemic symptoms (86\%). Major symptoms included inflammatory mass $(n=36$, $100 \%)$, abscess $(\mathrm{n}=33,92 \%)$, nipple discharge $(\mathrm{n}=7,19 \%)$, 

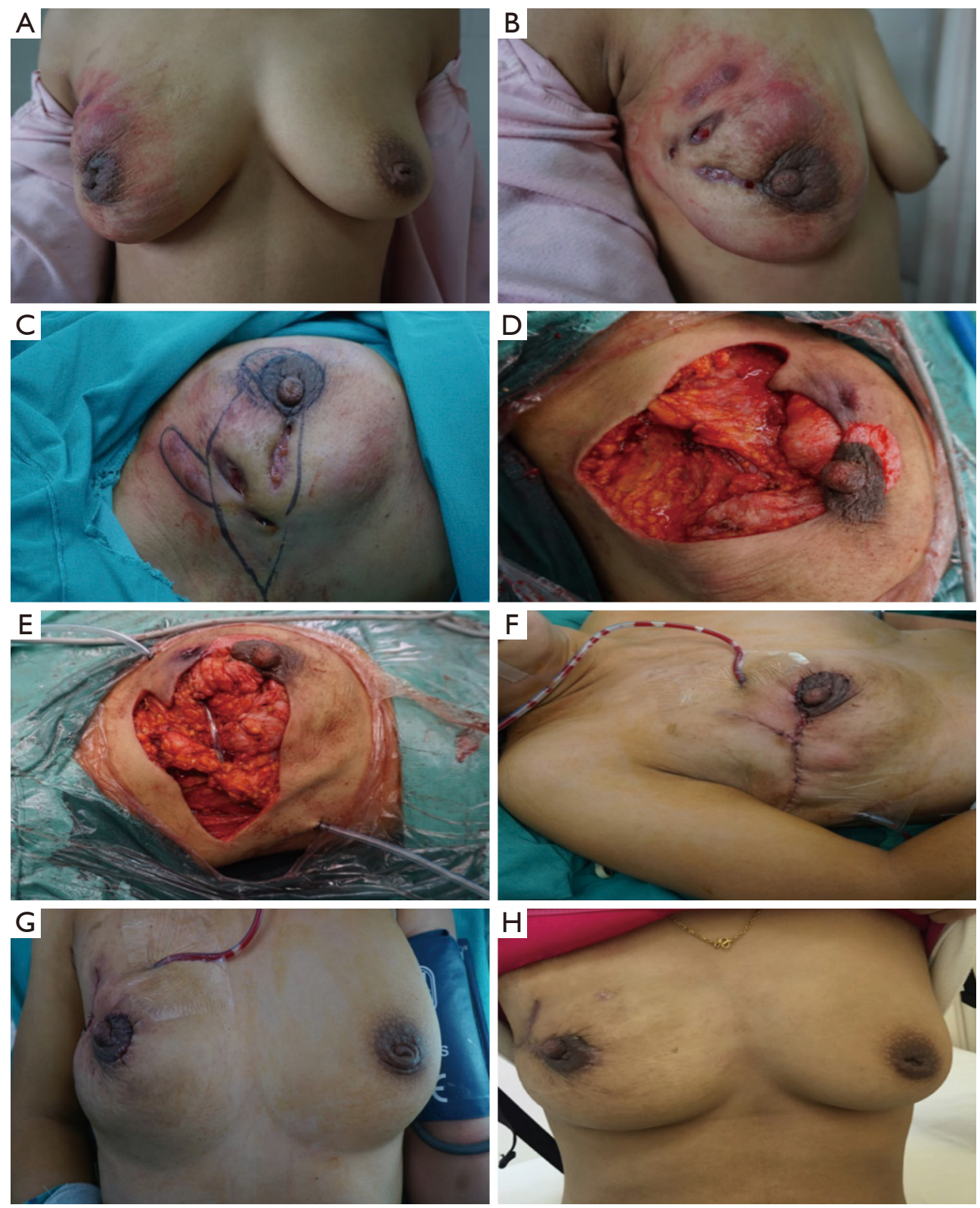

Figure 2 Continuous postoperative negative pressure irrigation assisted mammaplasty (CPNPIA) in treating chronic refractory plasma cell mastitis (CRPCM) in a 32-year-old patient. (A,B) Front and right side view of the patient's breast; (C) pre-operation, the dissection area was drawn on the skin; (D) all inflammatory masses, abscesses, sinus tract formations, and abnormal mammary ducts were completely removed; (E) a glandular flap originating from the surrounding breast tissue was separated and rotated to the excised area for immediate breast mammaplasty; (F) a negative pressure suction equipment was arranged into the surgical area for continuous postoperative negative pressure irrigation; $(\mathrm{G})$ the appearance of the breast on Day 7 after surgery; $(\mathrm{H})$ the appearance of the breast at 6-month follow-up.

inflammatory plaque ( $\mathrm{n}=34,94 \%)$, and sinus tract formation $(\mathrm{n}=19,53 \%)$ (Table 2). The lesion sizes ranged from 3 to $10 \mathrm{~cm}$ (mean $5.13 \mathrm{~cm}$ ) in diameter. The mean duration of the history of CRPCM before this surgery ranged from 1 to 13 months (mean 4.86 months). The mean duration of hospitalization was 8.42 days. The possible related risk factors for PCM included a congenital inverted nipple $(\mathrm{n}=13,36 \%)$, milk retention $(\mathrm{n}=7,19 \%)$, trauma $(\mathrm{n}=7$, $19 \%)$, and a history of breast surgery $(n=6,16 \%)$. No family history of PCM, autoimmune disease or endocrine 
hormone disorder was detected in these patients. None had received hormone replacement therapy, and all were non-smokers. Twenty patients displayed no related risk factors (Table 3). The success rate after surgery was $100 \%$ (36/36). Satisfaction ratings of breast shape after operation were "very good" ( $n=23,64 \%)$, "good" $(n=12,33 \%)$, and

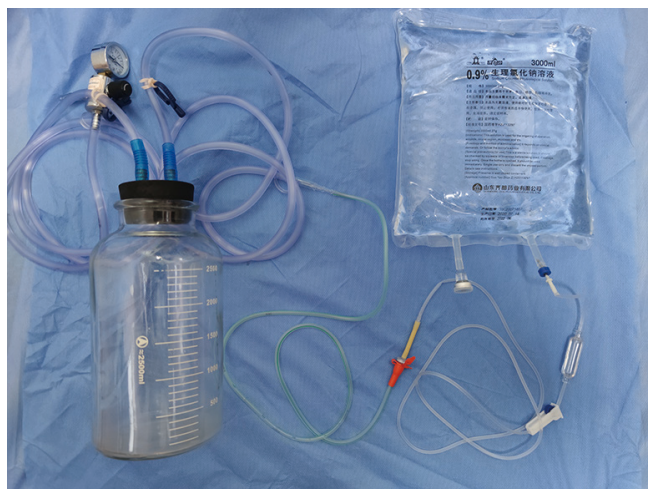

Figure 3 The equipment of continuous postoperative negative pressure irrigation includes saline irrigation, multiple-hole drainage tube and vacuum system. "moderate" ( $\mathrm{n}=1,3 \%)$. Satisfaction ratings of breast size were "very good" $(n=24,67 \%)$, "good" $(n=11,30 \%)$, and "moderate" $(\mathrm{n}=1,3 \%)$. Satisfaction ratings of breast symmetry were "very good" $(\mathrm{n}=19,53 \%)$, "good" $(\mathrm{n}=15$, $41 \%)$, and "moderate" $(n=2,6 \%)$. None of the patients had experienced a recurrence of symptoms at a 3 -month followup. The overall satisfaction in the procedure as rated by CRPCM patients was "very good" $(\mathrm{n}=22,61 \%)$, "good" $(\mathrm{n}=12,33 \%)$, and "moderate" ( $\mathrm{n}=2,6 \%)$, with no "poor" or "failed" ratings recorded in any category (Table 4).

\section{Discussion}

PCM is defined as a variant of aseptic mastitis. It is easily misdiagnosed and confused with other types of breast mastitis, including sarcoidosis, necrobiotic xanthogranulomatosis, fibrous mastitis, tuberculous granulomas, fat necrosis, and breast cancer (11). The etiology of PCM remains obscure. Ductal obstruction, hyperprolactinemia, autoimmune disease, infection, smoking and trauma are possible risk factors. In this study, the causes of PCM were as follows: a congenital inverted
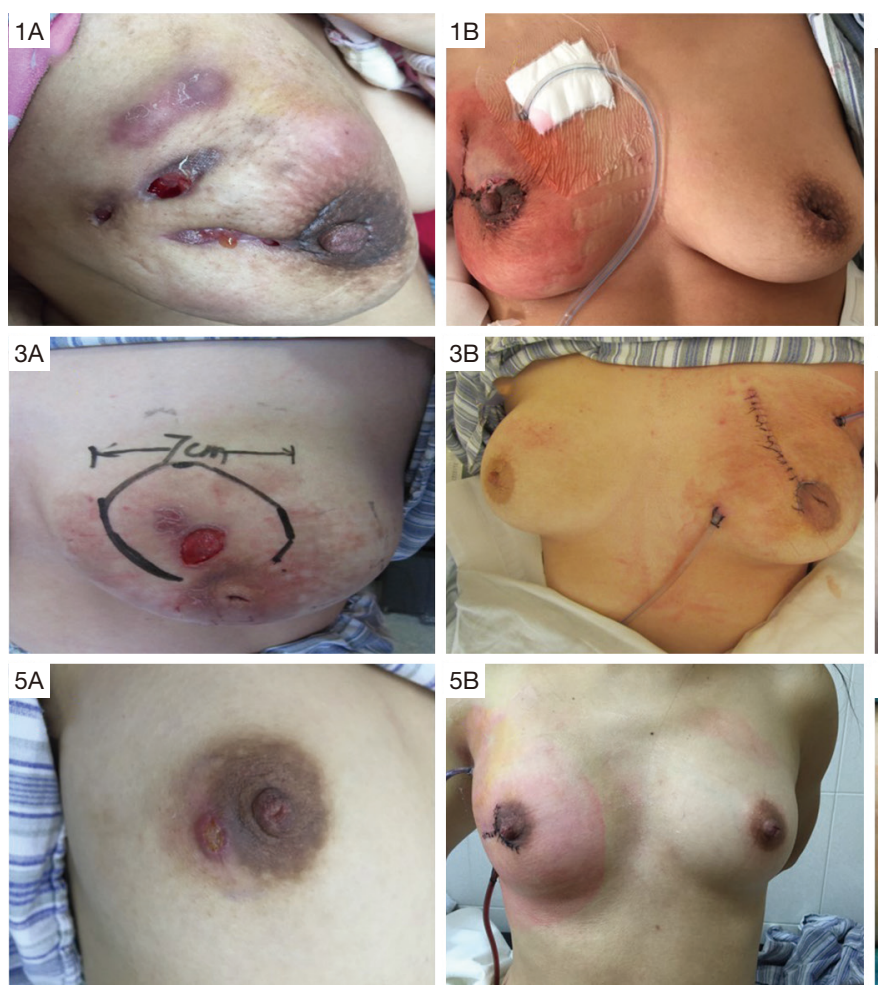
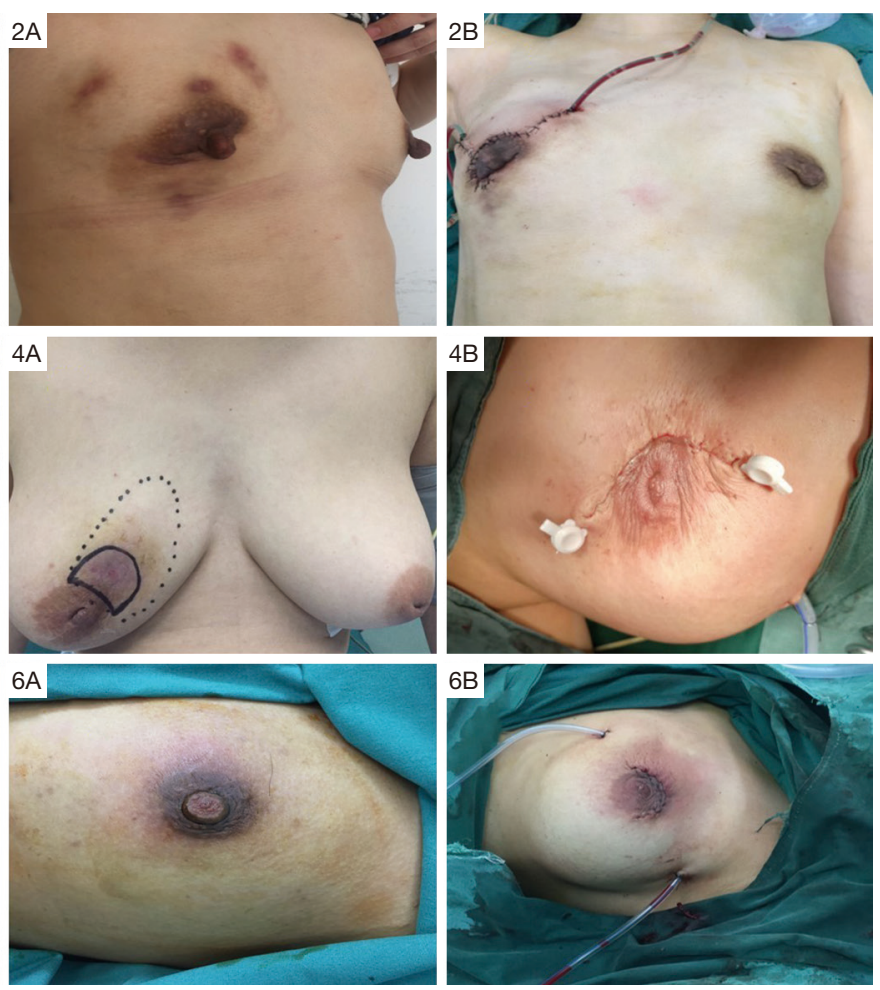

Figure 4 Images comparing the appearance and shape of the breasts before and after surgery. 1-6 indicate different patients, A indicates before surgery, and B indicates after surgery. 
Table 1 Distribution of patients by age ranges

\begin{tabular}{lccr}
\hline Age (years) & Numbers & Percentage (\%) & 20 \\
\hline $20-29$ & 10 & 28 & 20 \\
$30-39$ & 17 & 47 & 20 \\
$40-49$ & 6 & 17 & 210 \\
$50-59$ & 3 & 8 & 21 \\
$60-69$ & 0 & 0 & 21 \\
Total & 36 & 100 & 213 \\
\hline
\end{tabular}

Table 2 Symptoms of plasma cell mastitis (PCM)

\begin{tabular}{lccr}
\cline { 1 - 3 } Symptoms & Numbers & Percentage (\%) & 217 \\
\cline { 1 - 3 } Inflammatory mass & 36 & 100 & 219 \\
Abscess & 33 & 92 & 220 \\
Nipple discharge & 7 & 19 & 221 \\
Inflammatory plaque & 34 & 94 & 222 \\
Sinus tract formation & 19 & 53 & 223 \\
& & & 224
\end{tabular}

Table 3 Risk factor distribution

\begin{tabular}{lccc}
\cline { 1 - 3 } Risk factors & Numbers & Percentage (\%) & 228 \\
\cline { 1 - 3 } Congenital inverted nipple & 13 & 36 & 230 \\
Milk retention & 7 & 19 & 231 \\
Trauma & 7 & 19 & 232 \\
History of breast surgery & 6 & 16 & 233 \\
Autoimmune diseases & 0 & 0 & 234 \\
Hormone replacement therapy & 0 & 0 & 235 \\
Family history & 0 & 0 & 236 \\
Endocrine hormone disorders & 0 & 0 & 238 \\
History of smoking & 0 & 0 & 239 \\
No related risk factor & 20 & 56 & 240 \\
\hline
\end{tabular}

nipple, milk retention, trauma, and previous history of breast surgery. No patients had family history, autoimmune disease, endocrine hormone disorder, hormone replacement therapy, or smoking. The majority of the patients did not have any obvious trigger. Bani-Hani and Kessler showed that PCM normally occurred in young and middle-aged women who are not lactating or pregnant $(2,3)$. In our study, $75 \%$ of patients were also under 39 years old, and no patients were over 60 years old. Also, no patient was pregnant. The major symptoms of CRPCM included inflammatory mass, abscess, nipple discharge, inflammatory plaque, and sinus tract formation. Most of them presented inflammatory mass and abscess. The longest duration of CRPCM before surgery was 13 months. All patients in our study had received previous unsatisfactory conservative treatment or surgical treatment, such as antibiotics treatment, Chinese medicine treatment, repeated simple drainage or incomplete lumpectomy. The unsatisfactory treatment outcome, delayed healing process, and a high recurrence rate imposed physical and psychological burdens on the patients.

Surgical excision is a major advance in treating PCM $(12,13)$. Traditional surgery for PCM has typically included repeated simple drainage, lumpectomy, quadrant resection, or mastectomy $(4,14)$. Simple drainage is usually applied in CRPCM with abscess formation. Surgical processes include the complete opening of the abscess cavity, scraping of the necrotic tissue, and drainage. After simple drainage, the treatment cycle of patient can be lengthy and is often accompanied by pain, scar hyperplasia, breast deformity, and a high symptom recurrence rate. Lumpectomy and quadrant excision are also the most common surgical options, which have a high possibility of leading to recurrence and breast deformity. In some extreme cases, mastectomy is required when inflammation has invaded most of the breast tissue, and there is little normal gland remaining $(15,16)$. In our opinion, all inflammatory masses,

Table 4 Aesthetic outcome of plasma cell mastitis (PCM) comprehensive surgery

\begin{tabular}{lccccc}
\hline Variable & Very good & Good & Moderate & Poor & Failed \\
\hline Shape & $23(64 \%)$ & $12(33 \%)$ & $1(3 \%)$ & $0(0 \%)$ & $0(0 \%)$ \\
Size & $24(67 \%)$ & $11(30 \%)$ & $1(3 \%)$ & $0(0 \%)$ & $0(0 \%)$ \\
Symmetry & $19(53 \%)$ & $15(41 \%)$ & $2(6 \%)$ & $0(0 \%)$ & $0(0 \%)$ \\
Recurrence & $0(0 \%)$ & $0(0 \%)$ & $0(0 \%)$ & $0(0 \%)$ & $0(0 \%)$ \\
Overall & $22(61 \%)$ & $12(33 \%)$ & $2(6 \%)$ & $0(0 \%)$ & $0(0 \%)$ \\
\hline
\end{tabular}


abscesses, sinus tract formations, and abnormal mammary ducts should be thoroughly resected in once operation. The surrounding breast tissue should be carefully checked to ensure no lesions residue. Any remaining lesions may easily induce a recurrence or failure treatment of CRPCM.

Complete lesion excision could certainly lead to breast deformity. In our opinion, the best way to solve this aesthetic problem is to perform an immediate mammaplasty. Hladik reported using Deep Inferior Epigastric Perforator (DIEP) for breast reconstruction in three mastitis cases (15). However, infection of the surgical site after excision is a commonly reported complication. In our experience, the time-consuming surgery is not suitable for CRPCM cases because the prolonged surgical duration may significantly increase the risk of infection and a subsequent adverse outcome (9). We used glandular flaps originating from the surrounding breast tissue for immediate breast mammaplasty. The position of nipple areola was adjusted by removing dermis around areola and locally advanced skin flap was designed to cover the absence of skin, if necessary. The use of topical negative pressure therapy was first reported by Argenta and Morykwas in 1997 and was designed to stimulate granulation tissue formation and remove bacterial secretion (17). It is now widely practiced in treating acute and chronic wounds and surgical site inflammations (18-20). This study applied continuous postoperative negative pressure irrigation equipment, a modified negative pressure therapy system. The major advantage of this system is its effectiveness in diluting the pus and sucking away the necrotic tissue. The use of negative pressure drainage was effective in achieving complete wound closure, maintaining a sterile environment and preventing from secondary infection, which stimulated granulation tissue formation and accelerated the wound healing process.

There have been no standardized or systematic guidelines for diagnosing and treating PCM $(8,9)$. Treatment of CRPCM is complex due to the potential for surgical site infection, postoperative breast deformity, and a high recurrence rate. Therefore, the challenge for treating clinicians has been to find effective ways to shorten the disease course, decrease the recurrence rate, and reshape the breast. Traditional PCM surgical procedures involve repeated simple drainage, lumpectomy, quadrant resection or even mastectomy, and immediate breast mammaplasty was not recommended for infected breast. These surgical procedures may lead to scar hyperplasia, breast deformity or breast absence, and have a high recurrence rate. The advantage of CPNPIAM is that it enables the surgeons to 290 remove focuses and perform mammaplasty simultaneously, 291 which shortened the course of disease and maintained the shape of breast in one-step. The short-term follow-up data has revealed satisfying results in the success and recurrence rates and cosmetic appearance.

We acknowledge that there are limitations to this study. Larger sample sizes and cohort studies are necessary to assess this new surgical technique and confirm its clinical value in further study. Patients who received our treatment in this study suffered some mild physical inconvenience related to the CNPI procedure. Therefore, it will be important to consider ways to modify the drainage system and potentially shorten the treatment time in any future studies.

\section{Conclusions}

CPNPIAM provided a novel and effective way to treat patients with CRPCM and demonstrated a high success rate, a low recurrence rate, and a high satisfaction rating from patients. Larger sample sizes and multicenter randomized controlled studies are now necessary to verify the efficacy of this novel surgical technique in future research.

\section{Acknowledgments}

Funding: This work was supported by the Shanghai Municipal Commission of Science and Technology (No. 16401933200) and the National Natural Science Foundation of China, NSFC (No. 81503579).

\section{Footnote}

Reporting Checklist: The authors have completed the STROBE reporting checklist. Available at http://dx.doi. org/10.21037/gs-20-795

Data Sharing Statement: Available at http://dx.doi. org/10.21037/gs-20-795

Conflicts of Interest: All authors have completed the ICMJE uniform disclosure form (available at http://dx.doi. org/10.21037/gs-20-795). The authors have no conflicts of interest to declare.

Ethical Statement: The authors are accountable for all 337 
aspects of the work in ensuring that questions related to the accuracy or integrity of any part of the work are appropriately investigated and resolved. The study was approved by Shanghai Ninth People's Hospital, Shanghai Jiao Tong University School of Medicine institutional ethical committee standards (institutional ethical committee number: 2016-194-T138). All patients signed an informed consent form. The study conformed to the provisions of the Declaration of Helsinki (as revised in 2013).

Open Access Statement: This is an Open Access article distributed in accordance with the Creative Commons Attribution-NonCommercial-NoDerivs 4.0 International License (CC BY-NC-ND 4.0), which permits the noncommercial replication and distribution of the article with the strict proviso that no changes or edits are made and the original work is properly cited (including links to both the formal publication through the relevant DOI and the license). See: https://creativecommons.org/licenses/by-nc-nd/4.0/.

\section{References}

1. Cutler M. Plasma-cell mastitis: report of a case with bilateral involvement. Br Med J 1949;1:94-96.

2. Bani-Hani KE, Yaghan RJ, Matalka II, et al. Idiopathic granulomatous mastitis: time to avoid unnecessary mastectomies. Breast J 2004;10:318-22.

3. Kessler E, Wolloch Y. Granulomatous mastitis: a lesion clinically simulating carcinoma. Am J Clin Pathol 1972;58:642-6.

4. Lacambra M, Thai TA, Lam CC, et al. Granulomatous mastitis: the histological differentials. J Clin Pathol 2011;64:405-11.

5. Vinayagam R, Cox J, Webb L. Granulomatous Mastitis: A Spectrum of Disease. Breast Care (Basel) 2009;4:251-4.

6. Su L, Yu J, Liu C. Plasma-cell mastitis; report of 30 case with treatment of partial closure. Ning Xia Yi Ke Da Xue Xue Bao 2009;31:356-8.

7. Bundred NJ, Dover MS, Aluwihare N, et al. Smoking and periductal mastitis. BMJ 1993;307:772-3.

8. Ming J, Meng G, Yuan Q, et al. Clinical characteristics and surgical modality of plasma cell mastitis: analysis of 91

Cite this article as: $\mathrm{Xu} \mathrm{H}$, Jiang $\mathrm{Y}$, Liao M, Li D, Zhu C. Continuous postoperative negative pressure irrigation assisted mammaplasty in treating chronic refractory plasma cell mastitis. Gland Surg 2020;9(6):2071-2078. doi: 10.21037/gs-20-795 cases. Am Surg 2013;79:54-60.

9. Versluijs-Ossewaarde FN, Roumen RM, Goris RJ. Subareolar breast abscesses: characteristics and results of surgical treatment. Breast J 2005;11:179-82.

10. Panzacchi R, Gallo C, Fois F, et al. Primary sarcoidosis of the breast: case description and review of the literature. Pathologica 2010;102:104-7.

11. Cheng L, Reddy V, Solmos G, et al. Mastitis, a Radiographic, Clinical, and Histopathologic Review. Breast J 2015;21:403-9.

12. Akcan A, Akyildiz H, Deneme MA, et al. Granulomatous lobular mastitis: a complex diagnostic and therapeutic problem. World J Surg 2006;30:1403-9.

13. Raj N, Macmillan RD, Ellis IO, et al. Rheumatologists and breasts: immunosup-pressive therapy for granulomatous mastitis. Rheumatology (Oxford) 2004;43:1055-6.

14. Sabaté JM, Clotet M, Gomez A, et al. Radiologic evaluation of uncommon inflammatory and reactive breast Disorders. Radiographics 2005;25:411-24.

15. Hladik M, Schoeller T, Ensat F, et al. Idiopathic granulomatous mastitis: successful treatment by mastectomy and immediate breast reconstruction. J Plast Reconstr Aesthet Surg 2011;64:1604-7.

16. Yau FM, Macadam SA, Kuusk U, et al. The surgical management of granulomatous mastitis. Ann Plast Surg 2010;64:9-16.

17. Morykwas MJ, Argenta LC, Shelton-Brown EI, et al. Vacuum-assisted closure: a new method for wound control and treatment: animal studies and basic foundation. Ann Plast Surg 1997;38:553-62.

18. Malahias M, Hindocha S, Saedi F, et al. Topical negative pressure therapy: current concepts and practice. J Perioper Pract 2012;22:328-32.

19. Moisidis E, Heath T, Boorer C, et al. A prospective, blinded, randomized, controlled clinical trial of topical negative pressure use in skin grafting. Plast Reconstr Surg 2004;114:917-22.

20. Schwien T, Gilbert J, Lang C. Pressure ulcer prevalence and the role of negative pressure wound therapy in home health quality outcomes. Ostomy Wound Manage 2005;51:47-60. 